\title{
Ausencias en las primeras consultas de un centro de salud mental: un estudio controlado ${ }^{1}$
}

RESUMEN: Se analizan los factores que intervienen en las ausencias a las primeras consultas en un Centro de Salud Mental desde los tres grupos que los definen: atención primaria, el paciente y el centro de salud mental. La edad del paciente, la celeridad en la derivación y la lista de espera son las variables más importantes que influyen en este fenómeno

PALABRAS CLAVES: Ausencias. Primeras consultas programadas. Centro de Salud Mental.
SUMMARY: We analyse the factors involved in failure to keep initial appointments with Mental Health Centre. The analysis is divided in three parts: primary care, patients and the Mental Health Centre. Age of patients, referral timing (normal or preferential) and the length of time the patient waits are the most important factors.

KEY WORDS: Non-attendance. First appointment. Mental Health

\section{Introducción}

Las ausencias a las consultas programadas en Salud Mental es un problema asistencial que, además de poder incidir en la salud del paciente por su falta de atención, repercute en la pérdida de tiempo de los profesionales, el gasto económico para la institución y el aumento en la demora en la atención a aquellos pacientes que sí asisten. Las primeras citas se caracterizan por reservar un espacio de tiempo mayor en general, que permita llegar a una evaluación y orientación adecuada del paciente, por lo que la ausencia a este tipo de citas es especialmente gravosa. Teniendo en cuenta que el tiempo del profesional se paga con fondos públicos es importante que los implicados en este fenómeno mejoren la eficiencia de estas citas.

Las investigaciones realizadas sobre las ausencias en las primeras citas recogen tasas entre 10-60 \% (1-7). Estas investigaciones se han centrado principalmente en analizar las características personales, tanto clínicas como sociodemográficas, que puedan definir un perfil del paciente que no acude a estas primeras citas (1,3-11) y en valorar las estrategias preventivas que se pueden llevar a cabo para paliar este problema $(6,12-14)$. Todos estos trabajos difieren en la metodología porque ésta se ha empleado en función de los aspectos particulares que analizan en cada estudio. Por otro lado, la información que se tiene del paciente es más bien escasa y poco fiable porque no acude y esto complica el estudio de este fenómeno (6).

Los factores que inciden en la aparición de este problema se agrupan en tres vértices (2): el equipo de atención primaria (EAP) y las características de la derivación

${ }^{1}$ Una versión prelimiar y resumida de este trabajo recibió el primer premio compartido ex aequo al mejor póster en el VI Congreso de la Asociación Madrileña de Salud Mental celebrado el 6-7 de Junio de 2002.

Rev. Asoc. Esp. Neuropsiq., 2002, vol XXI, n. ${ }^{\circ}$ 83, pp. 27-36 
que realiza (motivo de la derivación, presión asistencial, mensaje que se le transmite al paciente...); los factores que tienen que ver con el paciente, sus características clínicas y sociodemográficas y, finalmente, las variables asociadas al Centro de Salud Mental (CSM) y que tienen que ver con su accesibilidad y la lista de espera.

Aunque algunos de los resultados son contradictorios, la mayor parte de los estudios no suele encontrar diferencias entre los ausentes y los que sí acuden a la consulta en las variables sociodemográficas salvo en la edad (1,3-10). Las características clínicas de los ausentes en las primeras citas corresponden a pacientes drogodependientes (3-6) o aquellos que presentan quejas vagas y poco específicas $(6,9)$ y suelen estar menos de acuerdo con la derivación que aquellos que, en contraste, acuden a petición propia y no suelen faltar $(1,7)$. Por último, una mala accesibilidad geográfica del CSM y la lista de espera más larga se correlacionan con un mayor porcentaje de ausencias $(1,3,4,6,11,15)$. Otros estudios han reflejado de forma anecdótica otros posibles factores influyentes como la hora de la cita (4), el profesional que va a atender al paciente (3), el hecho de que vaya a ser un residente (15) o el patrón estacional (4).

Los objetivos de nuestro estudio son hallar la prevalencia de ausencias en las primeras citas programadas en nuestro distrito, analizar los tres vértices del problema (paciente, centro de salud mental y atención primaria) en el grupo de pacientes ausentes y compararlo con un grupo control de pacientes que sí acudieron a la primera cita para verificar si existen diferencias significativas en dichos niveles.

\section{Material y métodos}

Diseño: Estudio observacional comparado aleatorizado.

Emplazamiento: CSM del distrito de Salamanca. Área 2 de Madrid.

Población: Nuestro CSM atiende a una población de 160.000 habitantes residentes en un distrito urbano de Madrid existiendo un predominio de la clase social media y media-alta.

Muestra: Un total de 1371 pacientes solicitaron primera cita en nuestro CSM en el período comprendido entre Febrero 2001- Febrero 2002. La derivación de los pacientes se realiza fundamentalmente desde atención primaria, y nos corresponden 7 equipos (E.A.P.)

Procedimiento: El médico de cabecera o, excepcionalmente, otro especialista rellena un volante de derivación que entrega en mano al paciente. El paciente tiene que acudir personalmente o a través de un familiar a pedir cita al CSM con el volante y le dan día y hora, aunque desconoce el profesional asignado. No se le vuelve a llamar para recordarle la cita. Se considera primera consulta la realizada por un paciente sin historia previa en nuestro CSM o, si la tienen, cuando han transcurrido más de 2 años desde que fueron atendidos por última vez. La lista de espera para derivaciones preferentes es habitualmente de menos de dos semanas, para derivaciones normales de alrededor de un mes si la consulta es con un psiquiatra y de unos 50-60 días si es con psicólogo. Este tiempo de espera cuenta desde que el paciente pide la cita, sin embargo, en este estudio lo contabilizamos a partir de la fecha en la que el médico firma el volante de derivación. El 
paciente puede tener el volante en su poder sin pedir cita en el CSM el tiempo que quiera por lo que la espera se puede incrementar en los resultados de este trabajo.

Variables y mediciones: A partir de los volantes de derivación y de las historias clínicas de aquellos pacientes que tenían antecedentes en nuestro CSM, se diseñó un protocolo de recogida de datos en los que se reflejaban las siguientes variables: sexo, edad, origen de la derivación, tipo de derivación (normal o preferente), E.A.P. y tiempo de espera a la primera consulta. Además, elegimos de forma aleatorizada a un número equivalente de pacientes que sí acudieron a su primera cita en nuestro CSM en el mismo período (grupo control) y lo comparamos con el grupo de ausentes para analizar si existen diferencias entre ambos.

Para poder estudiar las características clínicas de estos pacientes lo hemos hecho de forma indirecta de dos maneras. En los casos de pacientes con historia clínica se revisa el diagnóstico previo. Por otra parte, se analiza cuántos de los pacientes que fallan a su primera cita entre febrero y agosto de 2001 (105), vuelven a solicitar consulta en los siguientes seis meses, qué diagnóstico tienen si finalmente acuden a esta nueva primera cita (lo cual nos proporciona más datos clínicos para caracterizar a esta población), y cuál es su resolución (alta y derivación al EAP, seguimiento, abandono, alta voluntaria...) Se utilizó como prueba estadística el chi cuadrado tomando como significativo la $\mathrm{p}<0,05$.

\section{Resultados}

De un total de 1371 primeras citas programadas en nuestro CSM durante un período de 13 meses (febrero 2001-febrero 2002), 211 personas (15\%) no acudieron. La tabla 1 recoge los resultados de las variables estudiadas y su grado de significación en comparación con el grupo control.

Variables relacionadas con el paciente

La distribución por sexo es casi idéntica para ambos grupos: $65 \%$ de mujeres y $35 \%$ de hombres. La edad media del grupo de ausentes a la primera cita es de 40,1 años frente al grupo control que es de 43,8 años. Al estudiar el efecto conjunto de las variables sexo y no acudir a la primera cita sobre la edad (Tabla 2), vemos que hay una correlación de las ausencias con ser más joven, independientemente del sexo del paciente. Esta correlación entre edad y ausencias (Tabla 1) además es lineal y cuanto más mayor es el paciente, más acude a la primera cita (Gráfica 1).

Las características clínicas que definen a estos pacientes que no acuden a consulta las hemos estudiado indirectamente a través de los diagnósticos de los 17 pacientes que tenían historia previa en el CSM ( $8 \%$ del grupo de ausentes) y revisando las historias de $\operatorname{los} 14(13,3 \%)$ ausentes que en los seis meses siguientes (105 pacientes) podían pedir cita (Tabla 3). En el seguimiento, de los que vuelven a pedir cita en los 6 meses siguientes, casi un tercio vuelve a faltar y de los que acuden el $80 \%$ son dados de alta o abandonan tras la primera cita (Gráfica 2). Otro dato a destacar y que coincide con trabajos previos (1) es que los pacientes que tienen historia previa (y en nuestro caso llevan más de dos años sin contactar con el CSM) faltan ligeramente en menos ocasiones a la cita inicial actual (13\%). 
Variables relacionadas con la derivación

En relación con la fuente de derivación a salud mental, en ambos grupos el $90 \%$ de los casos son derivados desde Atención Primaria. Los porcentajes de derivación del resto de dispositivos (UHB/urgencias, especialistas, otros como servicios sociales, judiciales...) son similares también para los dos grupos y no hay diferencias significativas (Tabla 2).

Respecto a las derivaciones desde los Equipos de Atención Primaria, los resultados no muestran diferencias significativas entre ellos (Tabla 4). Por otro lado, sí observamos diferencias entre ambos grupos con respecto a la celeridad de la derivación: mientras que en el grupo de ausentes el 18\% de ellos son de carácter preferente, el grupo control estas derivaciones constituyen un $29 \%$, lo cual quiere decir que una mayor celeridad de la derivación, que conlleva a su vez un tiempo de espera menor, se asocia con un mayor cumplimiento en la primera cita (Tabla 1).

\section{Variables relacionadas con el CSM}

Nuestro CSM está ubicado en una zona céntrica de Madrid, situado en el borde del distrito al que atiende pero bien comunicado por transporte. Esto significa que la accesibilidad geográfica es buena $\mathrm{y}$, de hecho, no hay diferencias significativas en la procedencia de los E.A.P. de los ausentes a pesar de que la distancia a nuestro C.S.M. lógicamente no es igual (Tabla 4).

Por otro lado, la accesibilidad "burocrática" viene reflejada principalmente en el tiempo de espera para la primera cita. En un $30 \%$ de los casos, no hemos podido calcular el tiempo de espera ya que el médico no había registrado la fecha en el volante. Como el tiempo de espera (desde la fecha de la derivación hasta la primera consulta en el CSM) puede ser mayor que el real (desde que el paciente pide cita con el volante en el CSM, que no tiene por qué ser en la fecha de emisión del mismo, hasta la consulta) se explica que un $36 \%$ de los pacientes que se han ausentado esperen más de un mes cuando nuestro tiempo de espera suele ser menor. Si analizamos la distribución del tiempo de demora en periodos, podemos ver que el mayor porcentaje de ausencias se dan a partir de los 15 días (78\% del total), mientras que en el grupo control no presenta diferencias en los primeros 30 días aunque el porcentaje de los que acuden disminuye a partir de esta fecha (Tabla 1).

\section{Discusión}

No es la intención de nuestro estudio analizar todas las posibles variables que pueden estar implicadas en el fenómeno de las ausencias a la primera cita sino contrastar con otros trabajos aquellas que ya han mostrado ser las más importantes.

La incidencia de ausencias en la primera cita en nuestro CSM es del 15\%, cifra que está en el rango inferior de la mayoría de los estudios tanto en nuestro medio (Otero et al. 50\%, Herran et al. 17,5\%, Cáceres et al. 9,2\%, Livianos et al. 25\%) como fuera de España (Killapsy et al. 36\%, Neeleman et al. 21-33\%). 
Ningún estudio previo ha determinado un perfil sociodemográfico específico que discrimine a los pacientes que no acuden a la primera consulta en el CSM del resto de los pacientes que sí acuden. La característica más consistente que se repite en gran parte de los estudios es la edad, los pacientes más jóvenes son menos propensos que los más mayores a acudir a la primera cita (1,3-10). Nuestro trabajo corrobora este aspecto y la explicación al mismo tal vez pueda deberse a las obligaciones laborales de la población, ya que las ausencias disminuyen mucho a partir de los 50 años de edad media y este efecto es muy marcado a partir de los 65 cuando la población ya está jubilada. En este sentido hay que tener en cuenta que en nuestro CSM todas las consultas de pacientes nuevos se hacen en horario de mañana. No hay diferencias en cuanto al sexo, ya sea por la incorporación laboral de la mujer o por la dedicación a la casa y a los hijos de las mujeres jóvenes.

El estudio del perfil clínico de estos pacientes es difícil porque no van a consulta y porque los datos clínicos referidos en el volante de derivación muchas veces son inespecíficos, muy escuetos, o no reflejan el problema o la demanda real del paciente. Los estudios previos señalan que las drogodependencias, las quejas inespecíficas características de los trastornos de personalidad o los problemas sociales se asocian a un mayor número de ausencias a la primera cita(3-6). En nuestro trabajo, a través de los datos obtenidos en el seguimiento a los 6 meses y en las historias previas que son limitados, hemos visto que estos pacientes presentan, en su mayoría, trastornos menores de tipo neurótico o adaptativo y resulta excepcional encontrarse con pacientes más graves. Desde este punto de vista, faltarían a la primera consulta los pacientes leves, o aquellos que durante el tiempo de espera hasta la consulta mejoran lo suficiente como para no tener que acudir al especialista. Gran parte de las consultas en los CSM las originan pacientes con patología menor y, de hecho, en un estudio que realizamos en este mismo CSM hallamos que entre un $20-30 \%$ de los pacientes no presentan ningún trastorno mental diagnosticable (16), con lo cual no es de extrañar que muchos pacientes leves o no enfermos falten a la primera cita.

El proceso de derivación en el que se incluyen los criterios clínicos de la misma, el mensaje que se le da al paciente sobre su trastorno y las expectativas que se le crean, parece importante de cara a que el paciente acuda o no a la consulta en el CSM. Un hecho a tener en cuenta al estudiar las variables relacionadas con atención primaria es que en la actualidad, sólo mantenemos coordinación con uno de los EAP, Baviera, lo que podría influir en la forma y modo de derivación de los pacientes a salud mental. Es complicado poder analizar estas variables porque cada acto de derivación realizado por cada médico es único y fruto del encuentro que ha tenido con su paciente. De hecho, Killapsy (2000) señala cómo los ausentes a la primera cita en psiquiatría estaban menos de acuerdo con la derivación realizada por su médico de cabecera. Nosotros no hemos estudiado estas variables que definen cada proceso de derivación individual. Sin embargo, por encima de eso, sí que podría haber un "peso institucional" que determine en mayor o menor medida los criterios de derivación y la forma en que ésta se hace dependiendo de cómo entiendan la enfermedad mental y la relación que tengan con el CSM de referencia. En nuestro estudio no hemos hallado diferencias entre los distintos E.A.P. 
El modo de derivación, normal o preferente, es un mecanismo para asegurar que se atiende con prontitud a un paciente que lo precisa por su situación clínica. Muchas veces comprobamos que esa celeridad se basa en criterios administrativos (informes, bajas...) o en criterios clínicos discutibles. El paciente con un volante preferente tiene un tiempo de espera menor y por tanto se asocia con un mayor cumplimiento en la primera cita. En nuestro estudio hay una fuerte asociación en este sentido, pero llama también la atención que un $18 \%$ de las ausencias son derivadas preferentemente cuando al final estos pacientes no acuden a la cita y hemos visto que, en general, son leves. Este aspecto es importante por el agravio que supone para los pacientes más graves al prolongarse la lista de espera para pacientes preferentes de forma innecesaria.

El tiempo de espera parece ser una variable muy influyente en este fenómeno. Coincidiendo con la mayoría de los estudios (1,3-6, 13), el tiempo medio de demora en los ausentes es mayor (31,9 días) que en los asistentes (25,3 días). Si comparamos nuestros resultados con otros estudios donde su tiempo de espera es mayor al nuestro (1, 5), ésta es la variable más importante que marca la diferencia con sus hallazgos y que explica nuestro menor porcentaje de ausencias en la primera cita respecto a los suyos. Cabe la posibilidad de que los ausentes retengan más tiempo el volante de derivación antes de pedir cita, pero tres de los ocho E.A.P. comparten el edificio con el CSM y esto probablemente facilita que los pacientes pidan la cita el mismo día que les dan el volante.

Muchas de las investigaciones realizadas se han centrado en estudiar medidas preventivas que disminuyan este problema. En nuestro caso en concreto, contamos con la ventaja de que el tiempo de espera no es excesivo, exceptuando los casos de derivaciones normales para psicología, que podríamos intentar acortarlo. Tampoco cabe duda alguna que reportaría beneficios (no únicamente en este aspecto sino también en otros) mejorar la coordinación con atención primaria ya que aumentaría la calidad del proceso de derivación. Otras posibles estrategias que pueden paliar este problema asistencial se han estudiado en otros trabajos, como realizar avisos telefónicos antes de la primera cita (13), avisos por correo (17) o proporcionar cuestionarios al paciente cuando pida la cita para que los traiga rellenos en la primera consulta (18). Aunque parece que disminuyen la inasistencia a la primera cita, los resultados no son concluyentes (6).

\section{Conclusiones}

Las ausencias en las primeras consultas es un fenómeno universal de la realidad asistencial que conlleva costes económicos y de tiempo, pero que varía cuantitativamente entre unos dispositivos y otros y en nuestro CSM es del 15\%. Como en la mayoría de los estudios realizados, no existen diferencias significativas en relación con las características sociodemográficas excepto que los más jóvenes acuden menos a la primera cita. A diferencia de las ausencias en citas de seguimiento donde faltan en ocasiones pacientes graves, los pacientes que no acuden a la primera cita se corresponden en general con diagnósticos de trastornos mentales menores de corte neurótico y adaptativo. Aunque 
ORIGINALES Y REVISIONES

el proceso de derivación puede ser importante para que el paciente asista a nuestro CSM, no encontramos en nuestro estudio diferencias cuantitativas relevantes entre los distintos EAP. Aunque hay menos ausentes entre los pacientes preferentes, un $18 \%$ de estas ausencias fueron derivadas de forma preferente, lo cual supone un agravio para enfermos más graves al prolongarse la lista de espera de forma innecesaria. La lista de espera del CSM aparece como un factor muy importante en este fenómeno.

TABLA 1.- Comparación de los pacientes ausentes con el grupo control.

\begin{tabular}{|c|c|c|c|c|c|c|}
\hline & & \multicolumn{2}{|c|}{ Grupo Ausentes (211) } & \multicolumn{2}{|c|}{ Grupo Control (210) } & \multirow[b]{2}{*}{$\mathrm{p}$} \\
\hline & & $\mathrm{N}$ & $\%$ & $\mathrm{~N}$ & $\%$ & \\
\hline \multirow[t]{3}{*}{ Sexo } & Mujer & 138 & 65 & 137 & 45 & \\
\hline & Hombre & 73 & 35 & 73 & 35 & \\
\hline & $18-35$ & 105 & 50 & 88 & 42 & \\
\hline \multirow[t]{4}{*}{ Edad } & $36-50$ & 62 & 29 & 54 & 26 & $0,011^{*}$ \\
\hline & $51-65$ & 28 & 13 & 37 & 17 & \\
\hline & $>65$ & 12 & 6 & 31 & 15 & \\
\hline & EAP & 187 & 89 & 188 & 90 & \\
\hline \multirow{3}{*}{$\begin{array}{l}\text { Fuente de } \\
\text { derivación }\end{array}$} & Urgen/UHB & 14 & 7 & 10 & 5 & 0,698 \\
\hline & Especialista & 4 & 2 & 3 & 1 & \\
\hline & Otros & 6 & 3 & 9 & 4 & \\
\hline \multirow{3}{*}{$\begin{array}{l}\text { Tipo de } \\
\text { derivación }\end{array}$} & Normal & 174 & 82 & 149 & 71 & $0,0051^{*}$ \\
\hline & Preferente & 37 & 18 & 61 & 29 & \\
\hline & $<15$ días & 41 & 19 & 64 & 30 & \\
\hline \multirow{4}{*}{$\begin{array}{l}\text { Tiempo de } \\
\text { espera }\end{array}$} & 16-30 días & 75 & 35,5 & 62 & 30 & \\
\hline & 31-60 días & 56 & 26,5 & 43 & 20 & $0,014^{*}$ \\
\hline & $>60$ días & 8 & 4 & 6 & 3 & \\
\hline & Desconocido & 31 & 15 & 35 & 17 & \\
\hline
\end{tabular}

* Asociación estadísticamente significativa $\mathrm{p}<0,05$ 
TABLA 2.- Efecto conjunto de las variables sexo y ausencia sobre la edad media (SD)

\begin{tabular}{|l|c|c|c|}
\hline & Ausentes & Controles & \\
\hline Mujer & $\begin{array}{c}40,46 \\
(17,78)\end{array}$ & $\begin{array}{c}44,20 \\
(17,36)\end{array}$ & $\mathrm{F}=0,148$ \\
\hline Hombre & 39,63 & $\begin{array}{c}43,65 \\
(16,65)\end{array}$ & $\mathrm{p}=0,700$ \\
& $(16,80)$ & $\mathrm{F}=4,730$ & \\
\hline & \multicolumn{3}{|c|}{$\mathrm{p}=0,030$} \\
\hline
\end{tabular}

TABLA 3.- Diagnóstico de los ausentes con historia clínica previa y de los ausentes que piden nueva cita en los seis meses siguientes:

\begin{tabular}{|l|c|c|c|}
\hline & $\begin{array}{c}\text { Ausentes con } \\
\text { diagnóstico previo }\end{array}$ & $\begin{array}{c}\text { Ausentes que piden } \\
\text { nueva cita }\end{array}$ & Total \\
\hline Trastorno adaptativo & 8 & 3 & 11 \\
\hline Trastorno de ansiedad & 3 & 1 & 4 \\
\hline $\begin{array}{l}\text { Trastorno de } \\
\text { personalidad }\end{array}$ & 2 & 3 & 1 \\
\hline Trastorno sexual & 1 & & 1 \\
\hline Alcoholismo & 1 & 2 & 3 \\
\hline $\begin{array}{l}\text { Problemas familiares } \\
\text { o de pareja }\end{array}$ & 1 & 1 & 1 \\
\hline Esquizofrenia residual & & 10 & 1 \\
\hline Desconocido & 1 & & 27 \\
\hline Total & 17 & & \\
\hline
\end{tabular}

TABLA 4.- Total de derivaciones y ausencias por Equipos de Atención Primaria

\begin{tabular}{|l|c|c|c|c|}
\hline E.A.P. & Derivados & Ausentes & $\%$ & $\mathrm{p}$ \\
\hline Montesa & 241 & 33 & 13,6 & \\
\cline { 1 - 4 } Ramón de la Cruz & 281 & 44 & 15,6 & \\
\cline { 1 - 4 } Jorge Juan & 164 & 27 & 16,4 & \\
\cline { 1 - 4 } Baviera & 128 & 17 & 13,2 & \multirow{2}{*}{0,54} \\
\hline Castelló & 155 & 18 & 11,6 & \\
\hline Lagasca & 168 & 28 & 16,6 & \\
\hline Florestán Aguilar & 156 & 34 & 12,7 & \\
\hline Otros & 78 & 10 & 15,3 & \\
\hline Total & 1371 & 211 & \\
\hline
\end{tabular}


ORIGINALES Y REVISIONES

GRÁFICA 1.- Correlación entre la edad y la ausencia a las primeras consultas

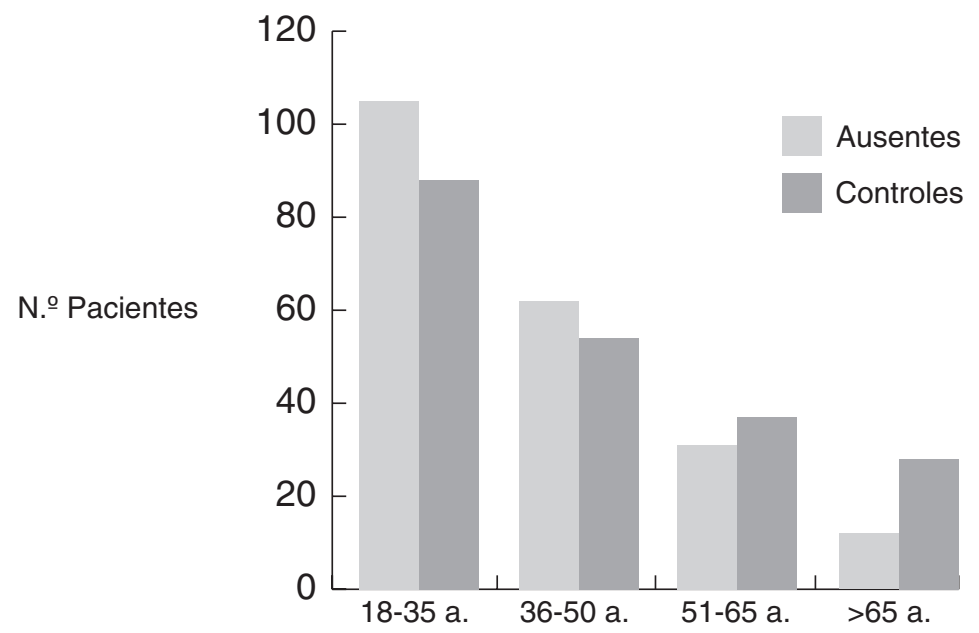

GRÁFICA 2.- Evaluación de ausentes que piden nueva consulta en los seis meses siguientes

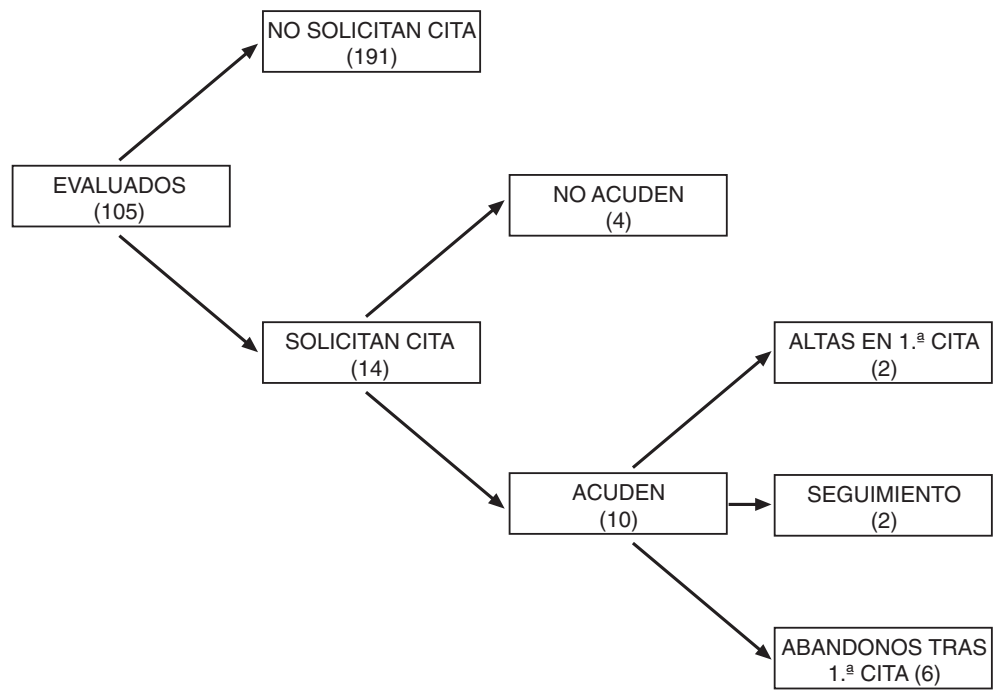

Agradecimientos: a Carolina Lozano Serrano por su colaboración en la recolección de los datos y a Francisco Rodríguez Salvanés por su ayuda en el análisis estadístico de los mismos. 


\section{BIBLIOGRAFÍA}

1. Otero J., Luque A., Conde M., Jiménez C., Serrano C. "Estudio de las variables asociadas a las ausencias en las primeras consultas psiquiátricas". Actas Esp. Psiq. 2001; 29(3):153-158.

2. Herrán A., López-Lauza J.R., Garizo H., Cadiñaños A., Díez-Manrique J.F., Vázquez-Barquero J.L. "Derivación de los pacientes con enfermedad mental desde Atención Primaria a Salud Mental". Actas Esp Psiq 2000; 28 (1):13-21.

3. Cáceres C., Osacar B. "Estudio de las variables que modulan la inasistencia a una primera consulta a un CSM". Anales de Psiquiatría 1994; 10(1):35-39.

4. Livianos-Aldana L., Vila-Gómez M., Rojo-Moreno L., Luengo-Gómez M.A. "Patients who miss initial appointment in community psychiatry? A Spanish community analysis". International Journal of Social Psychology 1999; 45 (3): 276-278.

5. Neeleman J., Mikhail W. "A case-control study of GP and patient-related variables associated with non-attendance at new psychiatric out-patient appointments". Journal of Mental Health 1997; 6 (3):301-306.

6. Nicholson Ph.D. "Factor involved in failure to keep initial appointments with mental health professionals". Hospital and Community Psychiatry 1994; 45 (3): 276-278.

7. Killapsy H, Banerjee S, King M, Lloyd M. "Prospective controlled study of psychiatric out-patient non-attendance" Br J Psychiatry 2000; 176:160-165

8. Raynes AE., Warren GH. "Some characteristics of dropouts at first contact with a psychiatric clinic". Community Mental Health Journal 1971; 7: 144-150.

9. Carpenter PJ., Morrow GR., Del Guardio AC., et al. "Who keeps the first appointment?" Am J Psych 1973; 130: 1157-1158.

10. Lowe RH. "Responding to "no-shows": some effects of follow-up method on community mental health center attendance patterns". J Consult Clin Psychol 1982; 50 (4): 602-603.

11. Orme Dr., Boswell D. "The pre-intake drop out at a community mental health center". Community Mental Health J. 1991; 27 (5): 375-379.

12. Kluger MP, Karras A. "Strategies for reduced missed initial appointments in a community mental health center". Community Mental Health J 1983; 19: 137-143.

13. Hochstadt NJ., Tribula J. "Reducing missed initial appointments in a community mental health center". J. Community Psychol 1980; 8: 261-265.

14. Swenson Tr., Pekan KG. "Interventions for reducing missed initial appointments in a community mental health center". Community Mental Health J 1988; 24: 205-210.

15. Campbell B., Staley D., Matas M. "Who misses appointments? An empirical analisis". Can J Psychiatry 1991; 36: 223-225.

16. Ortiz-Lobo A., García-Moratalla B., Mata-Ruiz I. "Las consultas de personas sin trastorno mental en un C. S. M". Ponencia en el VI Congreso de la Asociación Madrileña de Salud Mental, 6-7 de junio de 2002.

17. Kourany RFC, Garber J, Tornusciolo G. "Improving first attendance rates in child psychiatry outpatient clinics". Journal of the American Academy of child and adolescent psychiatry 1990;29:657-660

18. MacLean LM, Greenough T, Jorgenson V et al. "Getting trough the front door: improving initial appointment attendance at a medical health clinic". Canadian Journal of community Mental Health 1983;140:345-347

\footnotetext{
* Psiquiatra. Hospital Nuestra Sra. del Perpetuo Socorro. Albacete

** Psiquiatra. CSM del distrito de Salamanca. Área 2 de Madrid

Correspondencia: Alberto Ortiz Lobo. Centro de Salud Mental de Salamanca.

C/. O'Donnell, 55 - 28009-Madrid

Recibido: 28/08/02
} 